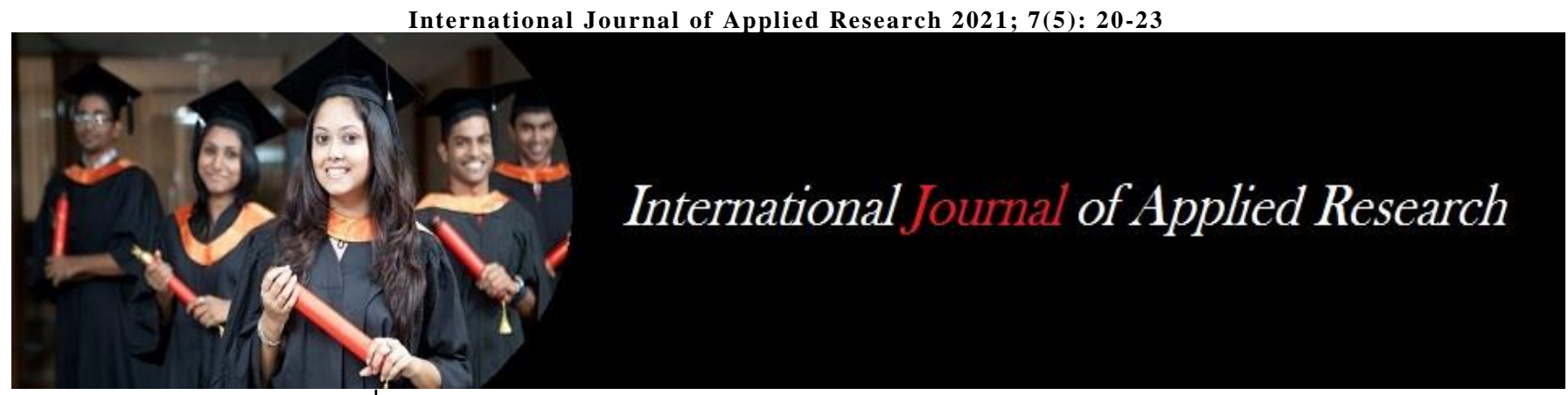

ISSN Print: 2394-7500 ISSN Online: 2394-5869 Impact Factor: 8.4

IJAR 2021; 7(5): 20-23 www.allresearchjournal.com

Received: 16-03-2021 Accepted: 18-04-2021

\section{Chandan Manna}

Ph.D. Research Scholar,

Department of Physical

Education and Sport Science,

Visva-Bharati, Santiniketan,

Birbhum, West Bengal, India

\section{Dr. Abhijit Thander}

Assistant Professor of Physical Education, Department of Physical Education and Sport Science, Visva-Bharati, Santiniketan, Birbhum, West Bengal, India
Corresponding Author: Chandan Manna Ph.D. Research Scholar, Department of Physical Education and Sport Science, Visva-Bharati, Santiniketan, Birbhum, West Bengal, India

\section{A comparative study of suryanamaskar and dynamic stretching on fast bowling performance in cricket}

\author{
Chandan Manna and Dr. Abhijit Thander
}

DOI: https://doi.org/10.22271/allresearch.2021.v7.i5a.8538

\begin{abstract}
The purpose of the present study was to compare the acute effects of suryanamaskar and dynamic stretching on the bowling performances of College Level Cricket Players. Thirty players competing in the District level and Club level Leagues took part in the study. The training protocols consisted of 10 mins suryanamaskar and Dynamic Stretching protocol was a series of specific progressive exercises lasting a total of 10 minutes over a distance of $20 \mathrm{mt}$. with a jog recovery (Young, 2007). The data collected on bowling accuracy and bowling speed without warm-up at baseline and immediately after the completion of two types of warm-up protocols namely Suryanamaskar and Dynamic Stretching were statistically analyzed by using a t-test. $\mathrm{P}$ value $<0.05$ was considered significant for this study. After the warm-up protocol, the effect of suryanamaskar and dynamic stretching has shown a high positive significant effect when compared to the baseline values of no warm-up protocol in eliciting the greatest performance in bowling accuracy and bowling speed. No significant difference was found after the warm-up protocols on bowling speed however bowling accuracy was found significantly different between the two groups. Based on the limitation of the study it can be concluded that both the protocol have a significant positive effect on bowling accuracy which can be used as warming up protocol.
\end{abstract}

Keywords: suryanamaskar, dynamic stretching, cricket bowling performance, fast bowling, cricket

\section{Introduction}

Universally the practice of warming-up has been accepted for a very long period. While the philosophy of warming up remains same, in recent years there is growing trends which questions the application of historic warm-up methods and bring the potential areas in practice for future development. A good warming up in fast bowling always help to maintain muscle balances, prevention of injury and improvement of bowling performance during a match and also allows a fast bowler to bowl fast because the bowlers are physically and mentally ready and reduce the risk of getting injured. McNair (2000) ${ }^{[7]}$ and Knudson (2001) [5] in a research study suggested the application of dynamic stretches where the slow controlled movements through the full range of motion are appropriate exercises during warm-up. On the other hand the incorporate of yoga now a days in cricket brings fruitful result. That's why the application of suryanamaskar as a warming up exercises as like dynamic stretching can be a good choice. Various research studies stated the benefits of suryanamaskar when it was applied as warming up protocol.

Basically literature shows that suryanamaskar stimulates large muscle group which stretches up to $97 \%$ of the body muscles and improves the fitness by strengthening the muscles (Gauri Shankar, 2011, Rajani Dalvi, 2012) ${ }^{[3,9]}$. Some research studies advocate a rapid method of suryanamaskar performing multiple rounds in a fast manner similar to physical exercise. It has been suggested that suryanamaskar at different speeds provides different benefits and that when it is done rapidly it warms up the body and acts as a cardio tonic, whereas when done slowly it strengthens and tones the musculature and enhances functioning of internal organs (Sun Salutation, 2010). Of these influencing outcomes, no research has been attempted to establish the application of suryanamaskar as like dynamic stretching the warmup session that's why the researchers were taken this study to find out by comparing the effectiveness of suryanamaskar and dynamic stretching on the bowling performances in cricket. 


\section{Methodology \\ Subjects}

Thirty (30) male cricket players who plays cricket in regular basis from West Bengal were selected as subjects for the study. The age of the subject ranged from 18-25 years. The subjects were categorized into two groups i.e. Dynamic stretching warm-up and Suryanamaskar. At the time of testing, all the subjects were bowled without being restricted by injury and all are "match fit".

\section{Criterion Measures}

The test items selected for this study were standardized and appropriate for the bowling performance on selected parameters like bowling accuracy and bowling speed which were measured by using the accuracy target Test in points and application of Kinovea software in meter/second (M/S) respectively.

\section{Research Design}

The experimental design was used in this study is the pretest-posttest design.

\section{Experimental Protocol}

The first 2 weeks of the study comprised 4 familiarization sessions ( 2 sessions per week) for subjects to become more comfortable and confident with Suryanamaskar and Dynamic Stretching protocol. Prior to the beginning of the experimental protocol subjects standing heights and weights were measured by using a stadiometer, weighing machine, and history of injury information were taken from the bowlers. For the purpose of the experimental protocol after completing normal warm-up procedures the bowlers of the two groups were given a protocol of dynamic stretching and Suryanamaskar practice for 10-12 mins. The selected variables namely bowling accuracy and bowling speed were measured before and after the experimental protocol.

\section{Training schedule}

\section{Group I (The Dynamic Warm-up (DW) group)}

In the dynamic warm-up protocols quadriceps, hamstrings, gastrocnemius, soleus, gluteus, adductors, and hip flexors mainly focused. The Dynamic Warm-up (DW) protocol used in the study was a Ten (10) minutes series of specific progressive exercises over a distance of twenty-meter with continues jogging recovery (Young, 2007)

\section{Group II (Fast Suryanamaskar group)}

Subjects went through a familiarization session to perform suryanamaskar by the researcher himself as he is a qualified yoga expert. All the Twelve (12) postures in suryanamaskar were performed in a rhythmic manner starting with an upright standing position and then moving into alternate forward and backward bending movements by involving both arms and legs and ended with an erect standing position. (Giri Swami Gitananda, 1981; Yogeswar, 1980) ${ }^{[4,}$ 11]

The subjects were trained to perform suryanamaskar in a rapid manner so that all Twelve (12) postures were completed in 2 minutes 6 rounds were performed in 10-12 minutes.

\section{Analysis and interpretation of data}

The data collected without warm-up at baseline and immediately after the completion of two types of warm-up protocols namely Suryanamaskar and Dynamic Stretching the Ball Accuracy and Bowling Speed were statistically analyzed by using a t-test. Intergroup comparison was analyzed with the paired t-test and intergroup comparison was analyzed by using an unpaired t-test. The significant level was set at 0.05 level for this study.

\section{Findings}

Mean and standard deviation of Personal data and anthropometric characteristics of the subject are tabulated and presented in the table 1 .

Table 1: Descriptive Statistical Parameters Of Personal Data And Anthropometric Characteristics Of The Subject

\begin{tabular}{|c|c|c|c|}
\hline \multirow{2}{*}{ Sl. No } & \multirow{2}{*}{ Variables } & SN Group & DS Group \\
\cline { 3 - 4 } & & Mean \pm SD & Mean \pm SD \\
\hline 1. & Age & $22.33 \pm 1.44$ & $22.866 \pm 1.457$ \\
\hline 2 & Height & $1.693 \pm 0.048$ & $1.684 \pm 0.028$ \\
\hline 3 & Weight & $58.933 \pm 6.307$ & $59.4 \pm 6.769$ \\
\hline 4 & Pace Bowling Category Slow-Medium & $27.915 \pm 1.738$ & $27.867 \pm 1.709$ \\
\hline
\end{tabular}

Table 2: Mean, Standard Deviation And T-Stat Of Bowling Performance Before And After The Warm-Up Protocols Of Suryanamaskar Group $(\mathbf{N}=\mathbf{3 0})$

\begin{tabular}{|c|c|c|c|c|}
\hline \multirow{2}{*}{ SI. No. } & \multirow{2}{*}{ Variables } & SN Group & SN Group & \multirow{2}{*}{ t-stat } \\
\cline { 3 - 4 } & & Mean \pm SD & Mean \pm SD & \\
\hline 1 & Ball Accuracy $($ points $)$ & $55 \pm 10.54$ & $64.16 \pm 10.54$ & $3.93^{*}$ \\
\hline 2 & Bowling Speed $(\mathrm{m} / \mathrm{s})$ & $27.832 \pm 1.963$ & $27.915 \pm 1.738$ & $4.63^{*}$ \\
\hline
\end{tabular}

*Significant at 0.05 level $(\mathrm{t}=2.16)$

Note: SN Group- Surya Namaskar Group; DS Group- Dynamic Stretching Group.

Table 2 reveals that the computation of 't' ratios of Suryanamaskar warming up group on bowling performance. The obtained' $t$ ' ratios on Ball accuracy and Bowling Speed were 3.93 and 4.63 respectively. Since these values were found to be higher than the required table value of 2.16, it was significant at 0.05 level of confidence. 
Table 3: Mean, Standard Deviation And T-Stat Of Bowling Performance Before And After The Warm-Up Protocols Of Dynamic Stretching Group $(\mathrm{N}=30)$

\begin{tabular}{|c|c|c|c|c|}
\hline \multirow{2}{*}{ Sl. No. } & \multirow{2}{*}{ Variables } & DS Group & DS Group & \multirow{2}{*}{ t-stat } \\
\cline { 3 - 5 } & & Mean \pm SD & Mean \pm SD & \\
\hline 1 & Ball Accuracy (points) & $57.6 \pm 25.814$ & $75.83 \pm 8.51$ & $2.24^{*}$ \\
\hline 2 & Bowling Speed (m/s) & $26.828 \pm 1.597$ & $27.867 \pm 1.709$ & $2.82^{*}$ \\
\hline
\end{tabular}

*Significant at 0.05 level $(\mathrm{t}=2.16)$

Note: FS Group- Fast Surya Namaskar Group; DS Group- Dynamic Stretching Group.

Table 3 reveals that the computation of 't' ratios of Dynamic warming up group on bowling performance. The obtained' $t$ ' ratios on Ball accuracy and Bowling Speed were 2.24 and
2.82 respectively. Since these values were found to be higher than the required table value of 2.16 , it was significant at 0.05 level of confidence.

Table 4: Comparison of Bowling Performance After Warm-Up Protocols Between the Suryanamaskar And Dynamic Stretching Groups $(\mathrm{N}=30)$

\begin{tabular}{|c|c|c|c|c|}
\hline \multirow{2}{*}{ Sl. No. } & \multirow{2}{*}{ Variables } & SN Group & DS Group & \multirow{2}{*}{ t-stat } \\
\cline { 3 - 4 } & & Mean \pm SD & Mean \pm SD & \\
\hline 1. & Ball Accuracy (points) & $64.16 \pm 10.54$ & $75.83 \pm 8.51$ & $3.156^{*}$ \\
\hline 2. & Bowling Speed $(\mathrm{m} / \mathrm{s})$ & $27.915 \pm 1.738$ & $27.867 \pm 1.709$ & 0.142 \\
\hline
\end{tabular}

*significant at 0.05 level $(\mathrm{t}=2.059)$

Table 4 reveals that the computation of 't' ratios of Suryanamaskar and Dynamic warming up group on bowling performance. The obtained ' $t$ ' ratio on Ball accuracy was 3.156. Since the value was found to be higher than the required table value of 2.059 , it was significant at 0.05 level of confidence.

\section{Discussion}

In the present study the warm-up protocols compared were Dynamic Stretching (DS) and Suryanamaskar (SN). However, this is the first study to investigate the effects of suryanamaskar and its application as an alternative protocol of stretching during warm-up.

The result of the present study shows that the bowling performance namely bowling speed and accuracy were enhanced from pre-test to post-test which seems that fast suryanamaskar and dynamic stretching warm-up protocol have significant effects on bowling performance. Because of limited studies on dynamic stretching and suryanamaskar and bowling performance a consensus statement regarding the effects of fast suryanamaskar and dynamic stretching warm-up protocol on pace bowling speed and accuracy is difficult to make from the current literature. It is unclear whether suryanamaskar and dynamic stretching improves pace bowling speed and accuracy, but it is notable that no study shows a performance benefit from the two types of warm up protocols performed prior to these bowling activities. However Faigenbaum et al. (2005) [1] and Yamaguchi and Ishii (2005) ${ }^{[10]}$ stated that that after dynamic type of stretching exercises force output increased which are happens by the augmentation of neuromuscular function, further the researchers stated that performance are highly effected by the dynamic type of stretching exercises because of its post activation potentiation effect via an proliferation in the rate of cross bridge attachments (1990) further Feros, S. A. (2015) ${ }^{[2]}$ in a study suggest that potentiation protocols could acutely enhance a bowler's speed capacity, and thereby allow a bowler to run-up with less effort and bowl with greater control. In the present study the enhancement of bowling performance might be due to potentiating effect of the two warm-up protocols.

In the present study the results did indicate that dynamic Stretching group has shown significantly better accuracy than the fast suryanamaskar group this may be due to the abilities of the pace bowlers under dynamic stretching group to keep the eyes on the desired target throughout the delivery stride and follow through for improved accuracy (Lillee, 1977) ${ }^{[6]}$ and the lower bowling speed may be a another factor which helps to maintain the good accuracy through-out the Spell. Further work is required to validate this accuracy protocol that simulates match demands.

Further, the result indicated that no significant differences were found after post-test when compared to the bowling speed between the two fast suryanamaskar and dynamic stretching group. The reason might be due to moderate exposure of the subjects in the competitive sport, further the insignificant difference in bowling speed might be due to homogeneity in experience level and personality characteristics, try to maintain their bowling accuracy throughout the spell or due to higher skill level.

\section{Conclusions}

Within the limitation \& findings of the study it may be concluded that bowling performance namely Accuracy and Bowling Speed were significantly altered after the suryanamaskar and dynamic stretching warm-up protocol. Bowling performance namely bowling Speed were found insignificant difference between the two groups after the warm-up protocols.

\section{References}

1. Faigenbaum AD, Bellucci M, Bernieri A, Bakker B, Hoorens K. Acute effects of different warm-up protocols on fitness performance in children. The Journal of Strength \& Conditioning Research 2005;19(2):376-381.

2. Feros SA. The Determinants and Development of Fast Bowling Performance in Cricket (Doctoral dissertation, Doctoral dissertation, Federation University Australia) 2015.

3. Gauri Shankar. The Effect of Suryanamaskar Yoga Practice on The Heart Rate, Blood Pressure, Flexibility and Upper Body Muscle Endurance in Healthy Adult. Bhavita Pancholi. International journal of health science $\&$ research, 2011, 1(1). 
4. Giri Swami Gitananda. Pondicherry: Satya press; Yoga: Stepby Step jumps on explosive force production and jumping performance. The Journal of Sports, 1981, 145-54.

5. Knudson D et al. Acute Effects of Stretching Are Not Evident in the Kinematics of the Vertical Jump, Journal of Strength \& Conditioning Research 2001;15(1):98101.

6. Lillee DK. The Art of Fast Bowling. Sydney, NSW: William Collins 1977.

7. Mcnair PJ et al. Stretching at the ankle joint: viscoelastic responses to holds and continuous passive motion. Medicine \& Science in Sport and Exercise 2000;33(3):354-358.

8. Medicine and Physical Fitness, 43(1):21-27

9. Rajani Dalvi. Surya Namaskar and holistic exercise. Indian streams Research journal 2012, 2(6).

10. Yamaguchi T, Ishii K. Effects of static stretching for 30 seconds and dynamic stretching on leg extension power. The Journal of Strength and Conditioning Research 2005;19(3):677-683.

11. Yogeswar Madras, Yoga Centre. 1st Ed. Mylapore: Textbook of Yoga 1980, 38-52.

12. Young WB, Behm DG. Effects of running, static stretching and practice jumps on explosive force production and jumping performance. The Journal of Sports Medicine and Physical Fitness 2003;43(1):2127. 